\title{
EXCLUSION OF AIR IN THE CULTIVATION OF THE GONOCOCCUS
}

\author{
E. H. R UEDIGER \\ From the Pathological Laboratory of the Bismarck Hospital, Bismarck, N. D.
}

During a general investigation on the cultural requirements of the gonococcus in which all aerobic methods proved more or less uncertain and unsatisfactory, exclusion of air was resorted to. A gramnegative diplococcus which did not grow on ordinary culture mediums, which did not grow on the special medium unless the air was excluded, and which was obtained from clinical gonorrhea was called gonococcus.

For the work $500 \mathrm{gm}$. of minced veal were boiled in a liter of distilled water for an hour. After cooling it was strained through cloth; with normal solution of sodium hydrate the filtrate was made neutral to phenolphthalein, and $15 \mathrm{gm}$. of bacto-agar added. After boiling until the agar was dissolved it was divided into 5 different portions - A, B, C, D and E. Portion A was tubed as it was. Portions $B, C, D$ and $E$ received bacto-peptone enough to make $1 \%$. In addition to peptone, portion $C$ received sodium chlorid enough to make $0.5 \%$; portion $D$ received glycerol enough to make $3 \%$ and portion $E$ received dextrose enough to make $1 \%$. The mediums were autoclaved in test tubes. After cooling sufficiently each portion was subdivided into portions 1 and 2 . Portion 1 received human blood which had been heated to about $56 \mathrm{C}$. for 30 minutes, and portion 2 received human blood which had not been heated. About $1 \mathrm{cc}$ of blood was added to $10 \mathrm{c} c$ of medium. The blood was well mixed with the medium, the tubes were placed in a slanting position and the medium allowed to solidify. After the medium had solidified each tube was stoppered air-tight with a sterilized cork stopper.

On comparing these different mediums it was found that the gonococcus did not grow well on those containing unheated blood.* It grew in all tubes which contained heated blood provided the stopper was inserted immediately after inoculation. Without the stopper growth did not take place as a rule. The largest growths - growths almost as luxuriant as those of typhoid bacilli on ordinary nutrient agar were obtained on portions B $1, C 1$ and D 1 , portion C 1 usually con. taining the best growth. Portion A 1 usually had a fair growth, whil portion $\mathrm{E} 1$ yielded poor growth.

$\mathrm{Up}$ to the present time the method has been used on three cases.

Received for publication, Dec. 18, 1918.

* Since this report was written very good subcultures have been obtained on mediu, containing unheated human blood. 
1. A young woman was operated on by Dr. N. O. Ramstad for a probable appendicitis. At operation acute right-sided salpingitis and local peritonitis was found. The right tube was removed. Some of the seropurulent contents were streaked on the various mediums. Growth occurred on all tubes except those which contained the unheated human blood. The best growths developed on tubes containing peptone, peptone and salt, and peptone and glycerol, the first two being superior to the last. Microscopic examination showed the growth to be a pure culture of a gram-negative diplococcus. The diplococcus did not grow on ordinary mediums, it did not grow on the special mediums without exclusion of air, it was obtained from clinically gonorrheal material, and hense was called gonococcus.

2. A woman giving a history of acute gonorrheal infection 3 months previous to examination was admitted to the hospital because of a painful, swollen knee. About $10 \mathrm{cc}$ of slightly turbid fluid were withdrawn from the knee joint by Dr. N. O. Ramstad. Some of the fluid was streaked out on the surface of the various mediums; growth occurred in all tubes which contained heated human serum. No growth appeared in the tubes containing unheated human serum. The growth in the tubes with heated human serum was composed of a gram-negative diplococcus somewhat irregular in size and shape; it did not brow on ordinary mediums; it did not grow on the special mediums without exclusion of the air; it was obtained from clinically gonorrheal material, and hence was called gonococcus.

3. A young man complaining of acute urethritis consulted Dr. Schoregge. The pus contained many characteristic, gram-negative intracellular diplococci. Some of the pus was streaked out on the surface of the various mediums. Growth appeared on all the mediums containing heated human serum; the best growths being on the mediums containing peptone, peptone and salt, and peptone and glycerol. The mediums with unheated human serum failed to show any growth. The growth which appeared on the medium with heated human blood was composed chiefly of diplococci, very few contaminating colonies being present. The diplococcus was irregular in shape and size, gram-negative, it did not grow on ordinary mediums nor on the special mediums without exclusion of the air; it was obtained from clinically gonorrheal material, and hence was called gonococcus.

\section{SUM MARY}

Cultures of gram-negative diplococci have been obtained from gonorrheal material on medium composed of veal broth, made neutral to phenolphthalein, agar, salt, peptone and $10 \%$ human blood which had been heated to $56 \mathrm{C}$. for 30 minutes. Little or no growth was obtained on medium contaiing unheated human blood. On medium without salt the diplococci grew about as well as on the medium with salt. The addition of glycerol or dextrose seemed to be unfavorable as was also the omission of peptone. The culture tubes had to be stoppered air-tight. The growth obtained was nearly as luxuriant as that of $B$. typhosus on ordinary nutrient agar and so far has been uniformly successful. 